\title{
CRITICAL DYNAMICS OF DIPOLAR FERROMAGNETS
}

\author{
E. FREY and F. SCHWABL \\ Institut für Theoretische Physik, Physik-Department der Technischen Universität München, D-8046 Garching, FRG
}

Received 10 March 1987; accepted for publication 13 May 1987

Communicated by A.A. Maradudin

\begin{abstract}
The dynamical scaling functions for ferromagnets with dipolar interactions are computed by mode coupling theory above the critical temperature $T_{c}$. On the basis of this theory we explain apparently conflicting features of neutron scattering experiments on EuO, EuS and Fe. The position of the crossover from isotropic to dipolar critical dynamics is determined and further experiments are proposed.
\end{abstract}

The dynamics of isotropic ferromagnets as EuO, EuS and Fe has been in focus ever since the very beginnings of the field of critical dynamics. Despite the large amount of experimental [1-6] and theoretical investigations $[7,8]$, there remain important open problems which have not been resolved up to now. (i) The expected crossover from isotropic to dipolar critical behaviour, accompanied by a change of the dynamical critical exponent $z$ from $5 / 2$ to 2 , has not been detected by neutron scattering experiments. (We disregard terms of order $\eta$.) (ii) The critical exponent deduced from neutron scattering data is $5 / 2$ [2-5]. But nevertheless the data could not be fitted by the Resibois-Piette [7] scaling function for isotropic ferromagnets [2]. (iii) A complete theory for the relevant scaling functions, which depend on two scaling fields, is still lacking. Hence the interpretation of the experimental data has been difficult if not impossible. Those apparent discrepancies remained a puzzle up to now and prompted a variety of interpretations including suggestions that dipolar forces might not be relevant for the dynamics of these magnets and that the data showed signatures of randomness and additional relaxation mechanisms.

In this paper we compute the dynamical scaling functions for weak dipolar ferromagnets. Our theory is based on the mode coupling theory, which has been very successful in pure isotropic ferromagnets. On the basis of our results we will be able to explain in a unifying fashion the apparently conflicting experimental results and will make further predictions for experiment.

The hamiltonian for a spin system with dipolar interactions is given by

$$
H=\int \frac{\mathrm{d}^{3} q}{(2 \pi)^{3}}\left(\left(\tau+J q^{2}\right) \delta_{i j}+J g \frac{q_{i} q_{j}}{q^{2}}\right) S_{i}(q) S_{j}(-q),
$$

where $S_{i}(q)$ is the Fourier transform of the cartesian components of the spin operator, $\tau$ a coefficient which does not enter explicitly in the equations of motion, $J$ the exchange interaction and

$$
g=a_{1}\left(g_{\mathrm{L}} \mu_{\mathrm{B}}\right)^{2} / 2 J a^{3}
$$

characterises the ratio of dipolar to exchange interactions. Here $a$ is the lattice constant and $g_{\mathrm{L}}$ the Lande factor. The coefficient $a_{1}$ depends on the lattice structure: $a_{1}=4 \pi(\mathrm{sc}), 3^{3 / 2} \pi(\mathrm{bcc}), 2^{5 / 2} \pi(\mathrm{fcc})$. The wave numbers are measured in units of $a^{-1}$. In eq. (1) we assumed that the dipolar forces are weaker than the exchange interaction [9]; i.e. $g \ll 1$.

Now we decompose $S(q)$ into a longitudinal and two transverse components 


$$
S(\boldsymbol{q})=S_{q}^{\mathrm{L}} \hat{q}+S_{q}^{\mathrm{T}_{1}} \hat{\boldsymbol{t}}^{1}(\hat{q})+S_{q}^{\mathrm{T}_{2}} \hat{\boldsymbol{t}}^{2}(\hat{\boldsymbol{q}})
$$

where

$$
\hat{\boldsymbol{q}}=q / q, \quad \hat{\boldsymbol{t}}^{1}(\hat{\boldsymbol{q}})=\boldsymbol{q} \times \boldsymbol{e}_{3} /\left(q_{1}^{2}+q_{2}^{2}\right)^{1 / 2}, \quad \hat{\boldsymbol{t}}^{2}(\hat{\boldsymbol{q}})=\hat{\boldsymbol{q}} \times \hat{\boldsymbol{t}}^{1}(\hat{\boldsymbol{q}}) .
$$

For vanishing components the limits are taken in the order of increasing cartesian components. The Heisenberg equations of motion are found to be

$$
\begin{aligned}
& \dot{S}_{q}^{\mathrm{L}}=J \int \frac{\mathrm{d}^{3} k}{(2 \pi)^{3}} \frac{1}{k}\left\{q(2 k-q)\left[\left(k_{1}^{2}+k_{2}^{2}\right)^{1 / 2}\left\{S_{q-k}^{\mathrm{T}_{1}}, S_{k}^{\mathrm{L}}\right\}+k_{3}\left\{S_{q-k}^{\mathrm{T}_{1}}, S_{k}^{\mathrm{T}_{2}}\right\}\right]+g\left(k_{1}^{2}+k_{2}^{2}\right)^{1 / 2}\left\{S_{q-k}^{\mathrm{T}_{1}}, S_{k}^{\mathrm{L}}\right\}\right\}, \\
& \dot{S}_{q}^{\mathrm{T}_{1}}=-J \int \frac{\mathrm{d}^{3} k}{(2 \pi)^{3}} \frac{1}{k}\left[q ( 2 k - q ) \left(\frac{-k_{1} k_{3}}{\left(k_{1}^{2}+k_{2}^{2}\right)^{1 / 2}}\left\{S_{q-k}^{\mathrm{T}_{1}}, S_{k}^{\mathrm{L}}\right\}+k_{1}\left\{S_{q-k}^{\mathrm{T}_{1}}, S_{k}^{\mathrm{T}_{2}}\right\}\right.\right. \\
& \left.\quad+\frac{k_{2}\left(k_{3} q-k^{2}\right)}{|q-k|\left(k_{1}^{2}+k_{2}^{2}\right)^{1 / 2}}\left\{S_{q-k}^{\mathrm{T}_{2}}, S_{k}^{\mathrm{L}}\right\}+\frac{1}{2} \frac{k_{2} q}{|q-k|}\left\{S_{q-k}^{\mathrm{L}}, S_{k}^{\mathrm{L}}\right\}-\frac{1}{2} \frac{k_{2} q}{|q-k|}\left\{S_{q-k}^{\mathrm{T}_{2}}, S_{k}^{\mathrm{T}_{2}}\right\}\right) \\
& \quad+g\left(\frac{-k_{1} k_{3}}{\left(k_{1}^{2}+k_{2}^{2}\right)^{1 / 2}}\left\{S_{q-k}^{\mathrm{T}_{1}}, S_{k}^{\mathrm{L}}\right\}+\frac{k_{2}\left(k_{3} q-k^{2}\right)}{|q-k|\left(k_{1}^{2}+k_{2}^{2}\right)^{1 / 2}}\left\{S_{q-k}^{\mathrm{T}_{2}}, S_{k}^{\mathrm{L}}\right)\right],
\end{aligned}
$$

and for $S_{q}^{T_{2}}$ correspondingly, where $\{$,$\} denotes the anti-commutator.$

The quatities of interest are the longitudinal and transverse Kubo relaxation funcitons

$$
\Phi^{\mathrm{L}(\mathrm{T})}(q, \omega, g)=\int_{0}^{\infty} \mathrm{d} t \mathrm{e}^{\mathrm{i} \omega t} \Phi\left(S_{q}^{\mathrm{L}\left(\mathrm{T}_{1}\right)^{\prime}}, S_{q}^{\mathrm{L}\left(\mathrm{T}_{1}\right)}, t\right)=\frac{\mathrm{i} \chi^{\mathrm{L}(\mathrm{T})}(q, g)}{\omega+\mathrm{i} \Gamma^{\mathrm{L}(\mathrm{T})}(q, \omega, g)},
$$

where

$$
\Phi(A, B, t)=\mathrm{i} \lim _{\epsilon \rightarrow 0} \int_{t}^{\infty} \mathrm{d} \tau \mathrm{e}^{-\epsilon \tau}\left\langle\left[A(\tau), B(0)^{\dagger}\right]\right\rangle .
$$

Here $\chi^{\mathrm{L}(\mathrm{T})}(q, g)$ are the longitudinal and transverse static susceptibilities and $\Gamma^{\mathrm{L}(\mathrm{T})}(q, \omega, g)$, the corresponding damping functions. Because of the rotational symmetry around the wave vector $q$ the correlation functions of $S_{q}^{\mathrm{T}_{1}}$ and $S_{q}^{\mathrm{T}_{2}}$ are equal.

Now we apply the standard procedure of mode coupling theory [11]: (i) We write down the Kubo formula for the transport coefficients $\Gamma^{\mathrm{L}(\mathrm{T})}(q, \omega, g)$. (ii) We consider two mode decay processes, which amounts to a factorisation of the Kubo formulas [12]. (iii) We make the lorentzian approximation; i.e. we replace $\Gamma^{\mathrm{L}(\mathrm{T})}(q$, $\omega, g)$ by $\Gamma^{\mathrm{L}(\mathrm{T})}(q, g)=\Gamma^{\mathrm{L}(\mathrm{T})}(q, 0, g)$. This leads to two coupled integral equations $(\alpha \equiv \mathrm{T}, \mathrm{L})$ :

$$
\begin{aligned}
& \Gamma^{\alpha}(q, g)=\frac{J^{2}}{\pi^{2} \chi^{\alpha}(q, g)} \int_{-1}^{1} \mathrm{~d} \eta \int_{0}^{\infty} \mathrm{d} k k^{2} \sum_{\beta} \sum_{\sigma} v_{\beta \sigma}^{\alpha}(k, q, g, \eta)\left(\delta_{\sigma, \mathrm{T}}+\delta_{\alpha, \mathrm{T}} \delta_{\beta, \mathrm{L}} \delta_{\sigma, \mathrm{L}}\right) \\
& \quad \times \frac{\chi^{\beta}(k, g) \chi^{\sigma}(|\boldsymbol{q}-\boldsymbol{k}|, g)}{\Gamma^{\beta}(k, g)+\Gamma^{\sigma}(|q-\boldsymbol{k}|, g)},
\end{aligned}
$$

where $\eta=\cos (k, q)$. The vertex functions $v_{\beta \sigma}^{\alpha}$ for the decay of the mode $\alpha$ into the modes $\beta$ and $\sigma$ are proportional to $\hat{v}_{\beta \sigma}^{\alpha}$, the scaled vertex functions defined by $v_{\beta \sigma}^{\alpha}=q^{4} \hat{v}_{\beta \sigma}^{\alpha}$. In an attempt to derive mode coupling equations for dipolar ferromagnets Borckmans et al. [8] used a decomposition of the spin operator (see eq. (2)) with one perpendicular direction $\boldsymbol{u}(\boldsymbol{q})$ only, which then is time dependent. Lacking an equation of motion 
Table 1

Asymptotic behaviour of the scaling functions.

\begin{tabular}{llllll}
\hline & DC & IC & DH & IH \\
\hline$\gamma^{\mathrm{T}}$ & $y^{1 / 2}$ & 1 & $y^{1 / 2} x^{2}$ & $x^{1 / 2}$ \\
$\gamma^{\mathrm{L}}$ & $y^{5 / 2}$ & 1 & $y^{5 / 2}$ & $x^{1 / 2}$ \\
\hline
\end{tabular}

for $u(q)$, the vertices in the mode coupling equations necessary for the computation of the scaling functions could not be determined.

As for the pure isotropic ferromagnet, the mode coupling equations do not account for effects of the critical exponent $\eta$, which will be neglected in the following. In the numerical calculations we will use the Ornstein-Zernike forms for the static susceptibilities

$$
\chi^{\alpha}(q, g)=J^{-1}\left(q^{2}+\xi^{-2}+\delta_{\alpha, \mathrm{L}} g\right)^{-1},
$$

where $\xi=\xi_{0}\left[\left(T-T_{\mathrm{c}}\right) / T_{\mathrm{c}}\right]^{-\nu}$. The static crossover is contained in $\xi$ through the effective exponent $\nu=\gamma_{\text {eff }} / 2$ [13].

The mode coupling equations (8) are consistent with the generalised dynamical scaling law

$$
\Gamma^{\alpha}(q, g)=\Lambda q^{z} \gamma^{\alpha}(x, y)
$$

introduced by Riedel and Wegner [12] for anisotropic magnets, where the scaling variables are

$$
x=1 / q \xi \text { and } y=g^{1 / 2} / q
$$

in the present case and $z=5 / 2$. The dynamical scaling functions for the line widths depend on two variables. They can be determined analytically from eqs. (6) in the dipolar (D) and isotropic (I) critical (C) and hydro-

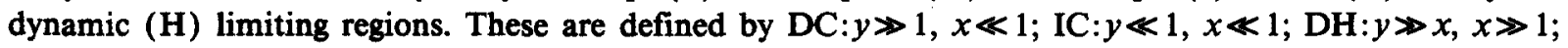
IH: $y \ll x, x \gg 1$. The results are summarised in table 1 .

Introducing eqs. (8) and (7) as well as

$$
\chi^{\alpha}(q, g)=J^{-1} q^{-2} \hat{\chi}^{\alpha}(x, y)
$$

into eq. (6), we find

$$
\begin{aligned}
& \gamma^{\alpha}(x, y)=\frac{2 \pi^{2}}{\hat{\chi}^{\alpha}(x, y)} \int_{-1}^{1} \mathrm{~d} \eta \int_{0}^{\infty} \mathrm{d} \rho \sum_{\beta} \sum_{\sigma} \hat{v}_{\beta \sigma}^{\alpha}(y, \rho, \eta)\left(\delta_{\sigma, \mathrm{T}}+\delta_{\alpha, \mathrm{T}} \delta_{\beta, \mathrm{L}} \delta_{\sigma, \mathrm{L}}\right) \\
& \times \frac{\rho_{-}^{-2} \hat{\chi}^{\beta}(x / \rho, y / \rho) \hat{\chi}^{\sigma}\left(x / \rho_{-}, y / \rho_{-}\right)}{\rho^{5 / 2} \gamma^{\beta}(x / \rho, y / \rho)+\rho_{-}^{5 / 2} \gamma^{\sigma}\left(x / \rho_{-}, y / \rho_{-}\right)}
\end{aligned}
$$

and the non-universal frequency scale of eq. (8)

$$
\Lambda=a^{5 / 2}\left(J k_{\mathrm{B}} T / 2 \pi^{4}\right)^{1 / 2}=g_{\mathrm{L}} \mu_{\mathrm{B}}\left(k_{\mathrm{B}} T a_{1} / 4 \pi^{4}\right)^{1 / 2} / q_{\mathrm{D}} .
$$

If $A$ is expressed by $q_{\mathrm{D}}=g^{1 / 2} / a$, the lattice structure dependence of $a_{1}$ has to be considered, which increases the theoretical $\Lambda$ of Fe by a factor of 1.14 . In eq. (10) we introduced $\rho=k / q, \rho_{-}=|q-k| / q, \eta=\cos (k, q)$ and the scaled vertex functions

$$
\begin{aligned}
& \hat{v}_{\beta \beta}^{\alpha}=\left[2 \eta^{2} \delta_{\alpha, \mathrm{L}}+\left(1-\eta^{2}\right)\left(\delta_{\beta, \mathrm{T}}+1 / 2 \rho_{-}^{-2}\right) \delta_{\alpha, \mathrm{T}}\right]\left(\rho \eta-\frac{1}{2}\right)^{2}, \\
& \hat{v}_{\mathrm{L}, \mathrm{T}}^{\alpha}=\left[2\left(1-\eta^{2} \delta_{\alpha, \mathrm{L}}\right)-\left(1-\eta^{2}\right)\left(1+1 / \rho_{-}^{-2}\right) \delta_{\alpha, \mathrm{T}}\right]\left(\rho \eta-\frac{1}{2}+\frac{1}{2} y^{2}\right)^{2} .
\end{aligned}
$$

For both longitudinal and transverse modes, the dipolar interaction enters only in decays into a longitudinal and a transverse mode. 


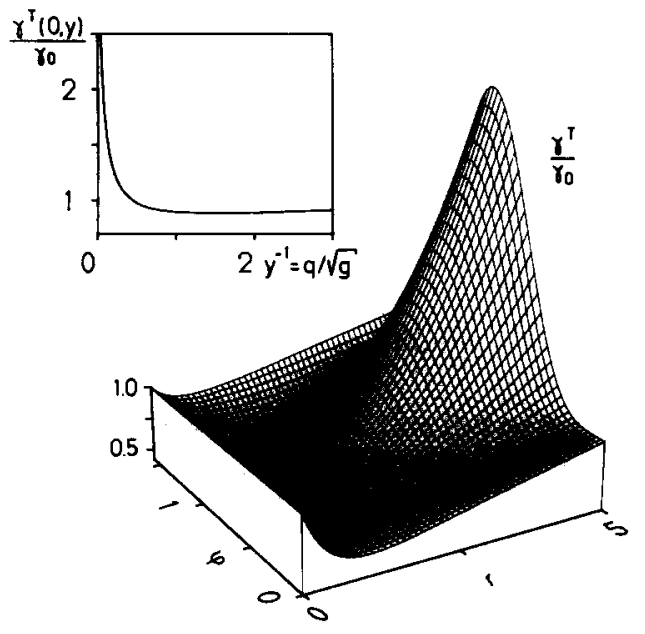

Fig. 1. Scaling function for transverse width $\gamma^{\mathrm{T}}$ versus $r=(q \xi)^{-1}\left(1+g \xi^{2}\right)^{1 / 2}$ and $\varphi=\operatorname{arctg}\left(g^{1 / 2} \xi\right)$. Inset: $\gamma^{\mathrm{T}}$ versus $q / g^{1 / 2}$ at the critical temperature $T_{\mathrm{c}}$.

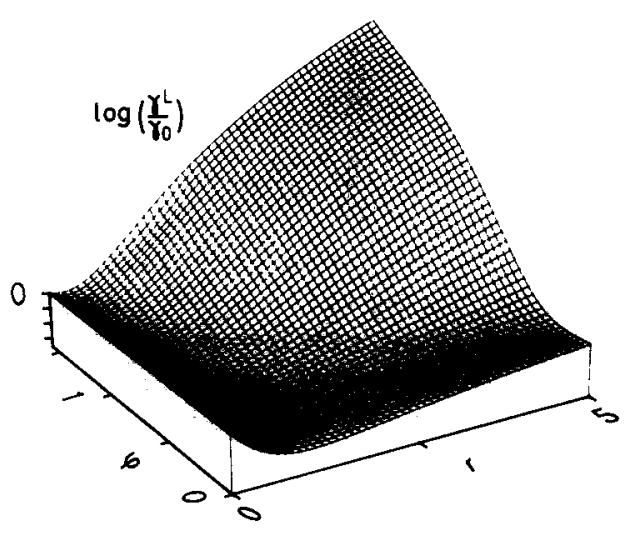

Fig. 2. Scaling function for longitudinal width $\gamma^{\mathrm{L}}$ versus $r$ and $\varphi$, defined in caption to fig. 1 .

For the numerical solution of the coupled integral equations $(10)$ we introduce polar coordinates

$$
r=\left(x^{2}+y^{2}\right)^{1 / 2} \text { and } \varphi=\operatorname{arct}(y / x) .
$$

The transverse and longitudinal scaling functions $\gamma^{\mathrm{T}}$ and $\gamma^{\mathrm{L}}$ are exhibited in figs. 1 and 2, in conformance with the analytic expressions of table 1 . All results are given in units of the value at criticality $\gamma_{0}=\gamma^{L(T)}(0,0)=5.1326$. In figs. 3 and 4 we display the scaling functions versus $x=1 / q \xi$ for different values of $\gamma=\operatorname{arctg}\left(g^{1 / 2} \xi\right)=N \pi / 40$ with $N=0,1, \ldots ., 19$. The curve $N=1$ is indistinguishable from the Resibois-Piette function, $N=0$. If $g$ is finite, the curves approach the Resibois-Piette function for small $x$ and deviate therefrom with increasing $x$. For a given material, $g$ is fixed and the parameterisation by $\varphi$ corresponds to a parameterisation by $T-T_{\mathrm{c}}$. The experimental results of Mezei on iron (fig. 3 of ref. [2] and fig. 4 of ref. [3]) show precisely the $(q \xi)^{-1}$ dependence for different temperatures as exhibited by the transverse scaling function (fig. 3). We are convinced that the uncertainty in the value of $\xi_{0}$, the improvement possible by taking into account the effective critical exponent

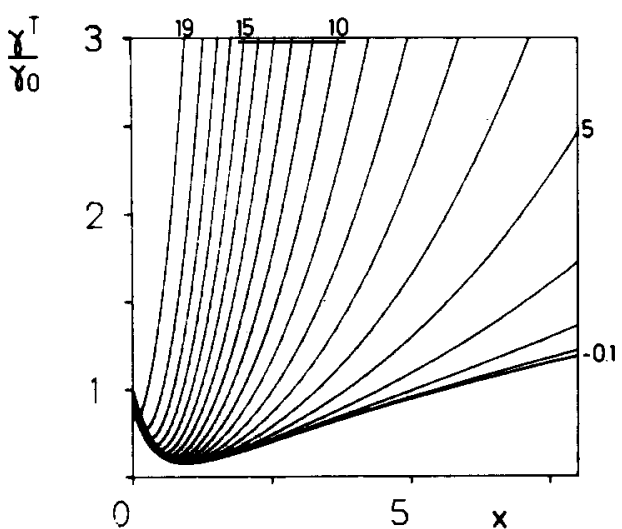

Fig. 3. Scaling function for transverse width $\gamma^{\mathrm{T}}$ versus $(q \xi)^{-1}$ for values of $\varphi=N \pi / 40$ with $N$ indicated in the graph.

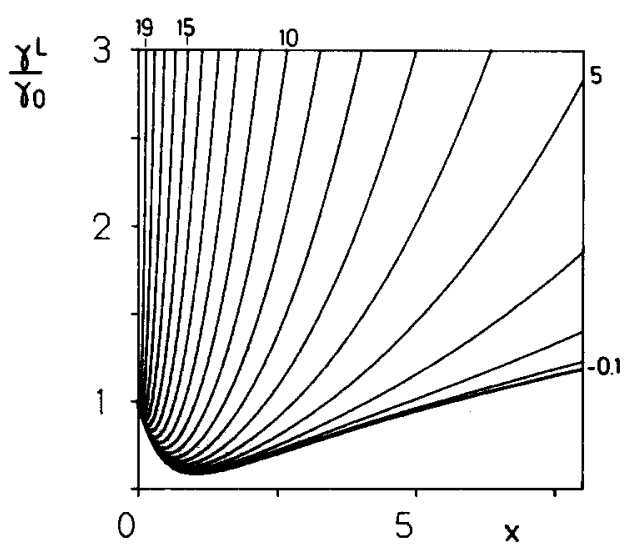

Fig. 4. Scaling function for longitudinal width $\varphi^{\mathrm{L}}$ versus $(g \xi)^{-1}$ for $\varphi=N \pi / 40$ with $N$ indicated in the graph. 
[13] $\nu \approx \gamma_{\text {eff }} / 2$ and the limits of the experimental accuracy, altogether leave enough room for a complete quantitative agreement with the theory.

In the inset to fig. 1 we display the transverse width against the wave number; i.e.: $y^{-1}=q / g^{1 / 2}$, precisely at the critical point. The most important conclusion to be drawn this graph is that the crossover from isotropic to dipolar critical dynamics occurs at a wave number smaller than $q_{\mathrm{D}}$, the position of the static crossover, by almost an order of magnitude. This explains why this crossover escaped the detection by neutron scattering experiments up to now. As matter of fact, there is an indication of an increase in the data with the smallest momentum transfer [6], as required by the theory.

In conclusion we note that the apparently contradicting experimental results can be explained in the framework of the present theory in the light of which the available data already give evidence for the expected dynamical isotropic-dipolar crossover. Certainly, real samples may contain imperfections giving rise to randomness; however, the purer the sample.the more it should obey the above theory. It should now be possible to reanalyse the experiments and investigate those areas where dipolar effects are predicted by the theory. An interesting check would be the measurement of the longitudinal width.

This work has been supported by the German Federal Minister for Research and Technology (BMFT) under contract number 03-I01A19-1.

\section{References}

[1] M.F. Collins, V.J. Minkiewicz, R. Nathans, L. Passell and G. Shirane, Phys. Rev. 179 (1969) 417; O.W. Dietrich, J. Als-Nielsen and L. Passell, Phys. Rev. B 14 (1976) 4923.

[2] F. Mezei, Phys. Rev. Lett. 49 (1982) 1096.

[3] F. Mezei, J. Magn. Magn. Mater. 45 (1984) 67.

[4] F. Mezei, Physica B 136 (1986) 417.

[5] P. Böni and G. Shirane, Phys. Rev. B 33 (1986) 3012.

[6] P. Böni, G. Shirane, H.G. Bohn and W. Zinn, preprint (1986).

[7] R. Resibois and C. Piette, Phys. Rev. Lett. 24 (1970) 514.

[8] P. Borckmans, D. Walgraef and G. Dewel, Physica A 91 (1977) 411

[9] A. Aharony and M.E. Fisher, Phys. Rev. B 8 (1973) 3323.

[10] K. Kawasaki, Prog. Theor. Phys. 39 (1986) 285; Ann. Phys. (NY) 61 (1970) 1; in: Phase transitions and critical phenomena, Vol. 5a, eds. C. Domb and M.S. Green, (Academic Press, New York, 1976).

[11] F. Schwabl, Z. Phys. 246 (1971) 13.

[12] E. Riedel and F. Wegner, Phys. Rev. Lett. 24 (1970) 730.

[13] A.D. Bruce, J.M. Kosterlitz and D.R. Nelson, J. Phys. C 9 (1976) 825. 